\title{
Arthrographic study of the rheumatoid knee. Part 2. Articular cartilage and menisci
}

\author{
KYOSUKE FUJIKAWA, YOSHINORI TANAKA, \\ TSUNEYO MATSUBAYASHI, AND FUJIO ISEKI
}

From the Department of Orthopaedic Surgery, School of Medicine, Keio University, 35 Shinano-machi, Shinjuku-ku, Tokyo, Japan

SUMMARY The changes of the articular cartilage and of the menisci in 129 knee joints with classical rheumatoid arthritis have been investigated by an improved arthrographic technique. The changes of the intra-articular components on the arthrograms coincided well with direct views at arthroscopy and surgery. The changes of the articular cartilage on the arthrograms were graded as normal, deposit and pooling, thinning, filling defect, destruction, and disappearance, and those of the menisci as normal, degeneration, tear, and disappearance. The results showed that: (1) The radiographs did not always reflect the changes of those intra-articular components in the early stages; especially in stage 2 they showed various conditions from intact to destruction. (2) The changes of the menisci were less advanced than those of the articular cartilage in the early stages. (3) The changes in those components progressed almost symmetrically in both compartments. It is necessary to appreciate not only the pathological condition of the bones of the knee joints but also that of the intra-articular components to devise more careful programmes of treatment for rheumatoid knees.

In general the intra-articular pathological conditions of the rheumatoid knee joint are evaluated by clinical findings and plain radiographs. However, it is difficult to appreciate the pathological conditions in the intra-articular components such as the articular cartilages, menisci, and ligaments on such information alone. Furthermore, since salvage operations are increasingly used for severely damaged rheumatoid knees, accurate information of the internal

Accepted for publication 20 August 1980

Correspondence to Dr K. Fujikawa, Rheumatism Research Unit, School of Medicine, University of Leeds, 36 Clarendon Road, Leeds LS2 9PJ. pathology is necessary. By improving the method of double contrast arthrography, ${ }^{1}$ it has become possible to apply this technique to rheumatoid knees.

In this paper the relationship between plain roentgenograms and the pathological conditions of the articular cartilages and menisci in 129 rheumatoid knee joints are discussed.

\section{Materials and methods}

This arthrographic study was undertaken on 129 knee joints of patients with classical rheumatoid arthritis according to the American Rheumatism

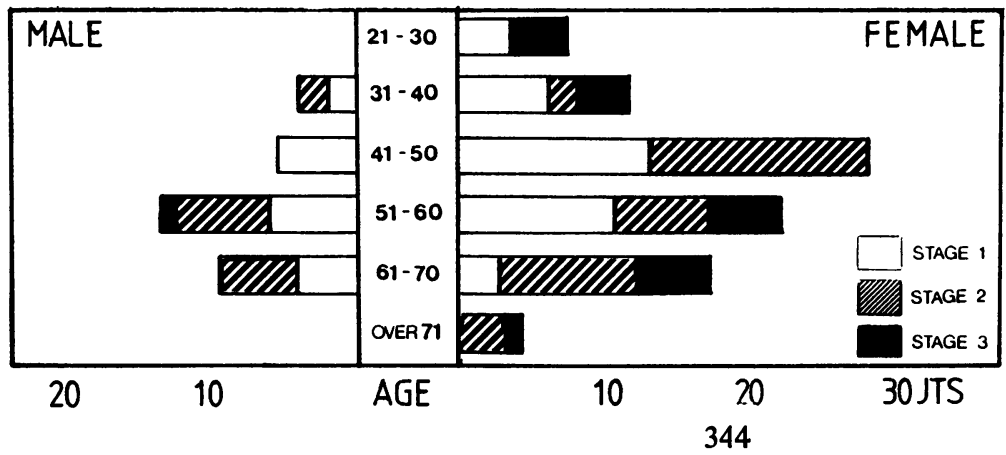

Fig. 1 Material. 
Association criteria (Fig. 1). Thirty-four were male joints and 95 female joints. The knee joints were divided into 3 groups by the roentgenographic classification of Steinbrocker. ${ }^{2}$ Stage 1 comprised 56 joints (male 18, female 38 ), stage 2, 53 joints (male 15 , female 38 ), and stage 3,20 joints (male 1, female
19). Most of these joints were examined periodically for several years by arthrography. The pathological condition of some of these joints was confirmed by direct vision at arthroscopy and/or surgery.

Arthrography as previously reported was used. ${ }^{\mathbf{3 4}}$ Four arthrograms, 2 anteroposterior views for the
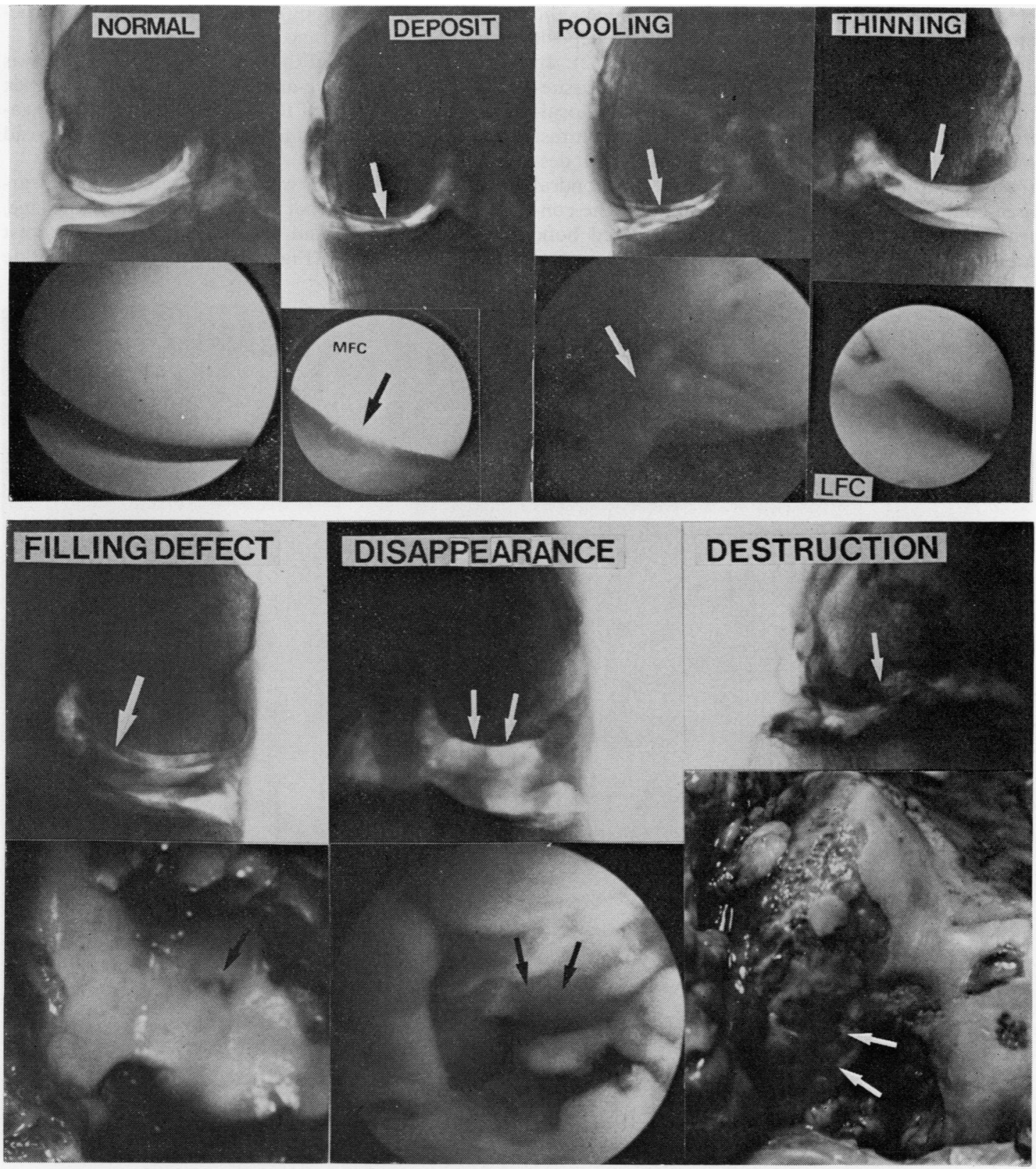

Fig. 2 Changes of articular cartilage on arthrograms of the knee with rheumatoid arthritis and relation to direct vision. 
lateral and the medial compartment, a lateral view, and a skyline (axial) view were routinely obtained. Evaluation of the plain roentgenograms was based on Steinbrocker's classification. ${ }^{2}$ The normal articular cartilage was displayed very smoothly and evenly, and the stains made by the contrast medium are thin and constant.

When the articular cartilage was eroded, the appearance became obscure with increasing deposit and pooling of the contrast medium (Fig. 2). In cases of ulceration by invasion of the synovial tissues the surface appeared abnormal as uneveness, localised thinning, and notable pooling of the contrast medium. In cases of advanced cartilagenous destruction a filling defect reaching to the subchondral area could be recognised. No deposition of the contrast medium could be seen on the exposed bone area.
These pathological changes of the articular cartilage on the arthrograms were graded as normal, deposit and pooling, thinning, filling defect, destruction, and disappearance; the findings correlated well with those seen at arthroscopy and/or surgery. They were categorised normal, slight change (deposit, pooling and/or thinning), moderate change (filling defect and/or thinning), and severe change (destruction and disappearance).

A cross-section of the corpus of the meniscus was imaged in an acute-angled triangle with its apex towards the centre of the joint. Some thin prolongations of the anterior and posterior horns were spread to the tibial spines.

When the menisci were degenerate, their appearance similar to that of the articular cartilage, became obscure, and the deposit of the contrast medium increased (Fig. 3). The severely degenerate

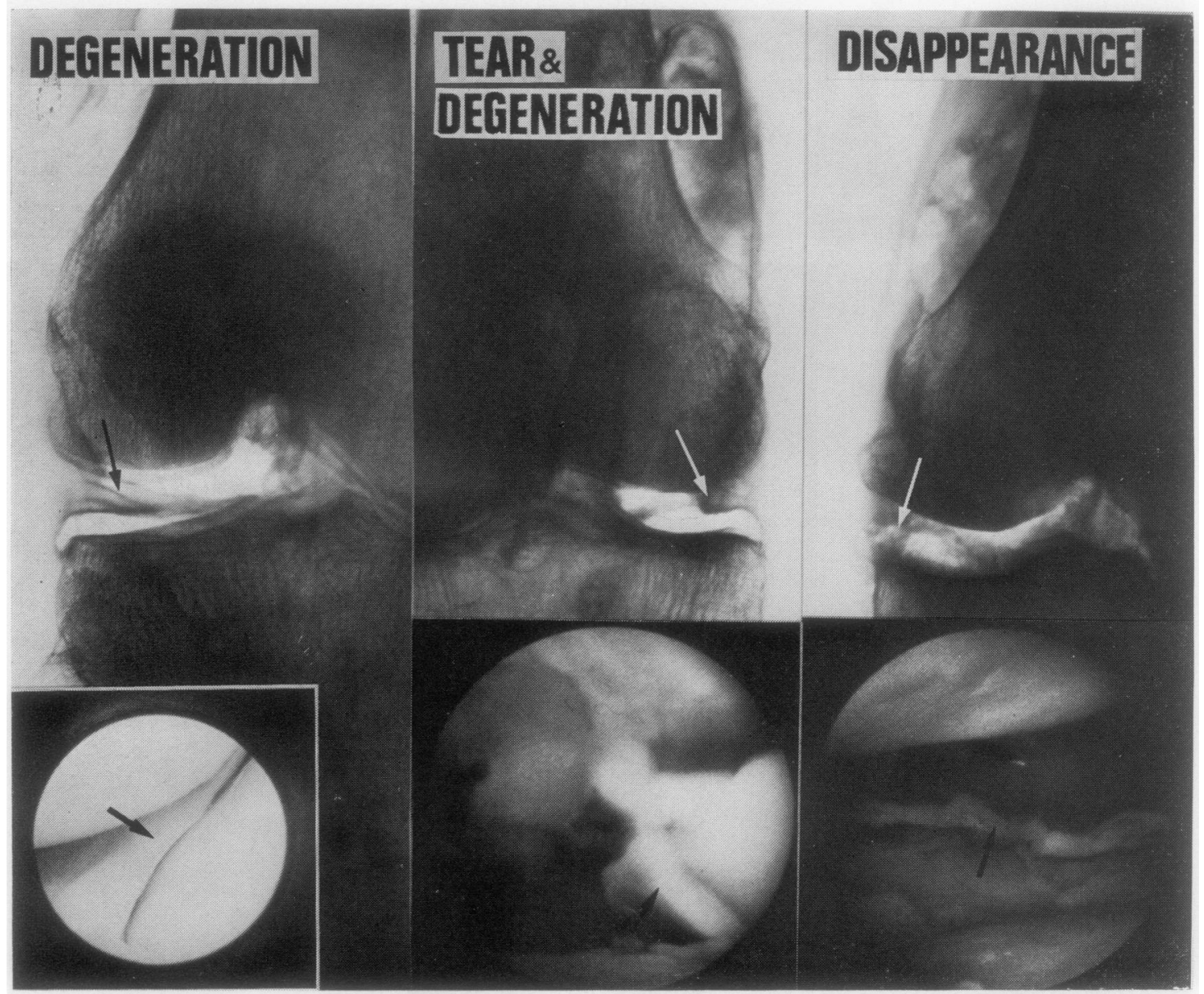

Fig. 3 Changes of menicsi on arthrograms of the knee with rheumatoid arthritis and relation to the direct vision. 
or torn menisci lost their original shapes by invasion of the contrast medium into the degenerated parts, fractures, and fissures. Those pathological changes of the menisci on the arthrograms were also evaluated as normal, degeneration, and tear and/or disappearance, which coincided well with direct views.

\section{Results}

\section{PLAIN ROENTGENOGRAMS}

Comparison of the stages of the medial and the lateral compartments of the rheumatoid knees showed no difference between these 2 compartments in $90.1 \%$ (Table 1 ). Of the remainder, in $9.1 \%$ the

Table 1 Relationship of the stage between medial compartment and lateral compartment of the tibio-femoral joint (numbers are percentages)

\begin{tabular}{llcl}
\hline Lateral & \multicolumn{2}{l}{ Medial } & \\
\cline { 2 - 4 } & Stage 1 & Stage 2 & Stage 3 \\
\hline Stage 1 & $46 \cdot 3$ & 7.4 & 0 \\
Stage 2 & 0 & 29.8 & 1.7 \\
Stage 3 & 0 & 0.8 & 14.0 \\
\hline
\end{tabular}

medial was more advanced than the lateral in its stage, and the reverse was found in only $0.8 \%$.

Joints with different stages in the medial and the lateral compartment were seen in the earlier stages. In the advanced stage, such as late stage 3 , most of the joints were at the same stage in both compartments. The pathological changes of the rheumatoid knee joints generally advanced at the same rate bilaterally and finally resulted in destruction.

\section{ARTICULAR CARTILAGE}

In stage 1 only $16 \cdot 1 \%$ showed no pathological change on the arthrograms; a slight change such as attrition and erosion could be seen in $78.5 \%$ and moderate change such as ulceration in $5.4 \%$ (Fig. 4). These changes in the articular cartilage were never demonstrated on plain radiographs.

Although in the stage 2 group the plain radiographs showed only slight change, by arthrography a moderate change of the articular cartilage could be seen in $26.7 \%$ and severe changes in $4.4 \%$. This means that one-third of the joints with a slight change on the plain radiographs had actually

\section{STAGE / CARTILAGE - 1}

\begin{tabular}{|c|c|c|c|c|}
\hline \multicolumn{2}{|c|}{\begin{tabular}{|l|l|} 
CHANGE & STAGE \\
OF CARTILAGE & \\
\end{tabular}} & $\begin{array}{c}1 \\
(56 \mathrm{JTS}) \\
\end{array}$ & $\left(45^{2}\right.$ JTS.) & $\left.{ }^{3}{ }^{3} \mathrm{JTS}\right)$ \\
\hline \multicolumn{2}{|c|}{ NORMAL } & 16.1 & 2.2 & \\
\hline \multirow{2}{*}{$\begin{array}{l}\text { SLIGHT } \\
\text { CHANGES }\end{array}$} & DEPOSIT & 37.5 & 8.9 & \\
\hline & $\begin{array}{l}\text { POOUNG ANDIOR } \\
\text { THINNING }\end{array}$ & 41.0 & 57.8 & \\
\hline \begin{tabular}{|c|} 
MODERATE \\
CHANGES \\
\end{tabular} & $\begin{array}{c}\text { FILLING } \\
\text { DEFECT } \\
\end{array}$ & 5.4 & 26.7 & \\
\hline \multirow{2}{*}{$\begin{array}{l}\text { SEVERE } \\
\text { CHANGES }\end{array}$} & DESTRUCTION & & 4 & \\
\hline & DISAPPEARING & & & \\
\hline
\end{tabular}

Fig. 4(a) Relationship between stage and changes of articular cartilage-1.

\section{STAGE I CARTILAGE - 2}

\begin{tabular}{|c|c|c|c|}
\hline $\begin{array}{l}\text { CHANCES STAGE } \\
\text { OF CARTLAGE }\end{array}$ & $\begin{array}{c}1 \\
\text { (56 JTS.) }\end{array}$ & $\begin{array}{c}2 \\
\text { ( } 45 \text { JTS) }\end{array}$ & $\frac{3}{\left(20^{\mathrm{JTS}}\right)}$ \\
\hline NORMAL & 16.1 & 2.2 & \\
\hline SLIGHT & & 66.7 & \\
\hline MODERATE & & 26.7 & \\
\hline SEVERE & & 4.4 & \\
\hline
\end{tabular}

Fig. 4(b) Relationship between stage and changes of articular cartilage-2. 
STAGE IMENISCUS

\begin{tabular}{|c|c|c|c|c|c|c|}
\hline \multicolumn{3}{|c|}{ LATERAL } & \multirow{2}{*}{$\begin{array}{l}\text { CHANGE } \\
\text { MENISCUS }\end{array}$} & \multicolumn{3}{|c|}{ MEDIAL } \\
\hline STAGE 3 & STAGE 2 & STAGE 1 & & STAGE 1 & STAGE 2 & STAGE 3 \\
\hline & 22.2 & S71.4 & NO CHANGE & 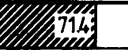 & 22.2 & \\
\hline 5.0 & 60.0 & 23.2 & DEGENERATION & 28.6 & 69.0 & 10.0 \\
\hline 5.0 & 13.3 & 5.4 & TEAR & & 2.2 & 5.0 \\
\hline 800 & & & DSAPPEARANCE & & 22 & 850 \\
\hline 10.0 & 4.5目 & & UNDETECTED & & 4.4 & \\
\hline
\end{tabular}

Fig. 5 Relationship between stage and changes of menisci.

already advanced to a moderate stage or beyond. In the stage 3 group all joints showed severe change, and the articular cartilage had already vanished in up to $95 \%$ on the arthrograms. It is apparent that plain radiographs do not always express the pathological changes of the intra-articular components well, particularly in the stage 2 group, which is the most important clinically.

\section{MENISCI}

The changes of the menisci in the rheumatoid knees were almost equal in the medial and lateral compartment. This pattern differed greatly from that of the osteoarthritic joint, in which the medial meniscus is more severely damaged than the lateral in general. It was similar to the articular cartilage in that the pathological changes of the medial became more severe as the stage advanced.

In stage 1 there were no abnormal findings on the arthrograms in $71.4 \%$ of the medial and the lateral menisci. A slight change, mainly degeneration, could be seen in $28.6 \%$ of the medial and $23.2 \%$ of the lateral. A torn meniscus was recognised only in $5.4 \%$ laterally and a severe change such as disapperance was never seen in the stage 1 group.

In stage 2 the proportion of the normal menisci decreased to $22.2 \%$ on both sides. The degenerative changes of the menisci increased up to $69.0 \%$ in the medial $60.9 \%$ in the lateral. Torn menisci occurred in $2.2 \%$ of the medial and $13.3 \%$ of the lateral. Though torn menisci could be observed more frequently laterally, disappearance of the menisci, a much more severe change, was recognised only medially. In stage 3 all joints showed changes in the menisci. Only $5 \%$ of the medial menisci and $10 \%$ of the lateral showed slight change, and in $5 \%$ of each side torn menisci could be observed. The medial menisci had already disappeared in $85 \%$ and the lateral in $80 \%$. Most of the menisci, therefore, at this stage showed severe changes.

These results suggest that plain radiographic findings do not always reflect the pathological change in the menisci, particularly at stage 2 .

\section{Discussion}

Pathological changes in the rheumatoid knee joint usually begin from synovial tissue and extend to the articular components such as the cartilage. menisci, and ligaments until the joint is destroyed completely. There is no doubt that the destruction of the cartilage especially influences the prognosis of the joint function.

As Steinbrocker's classification of the stage from plain radiographs is not only simple but easy to apply, it has been popular in the evaluation of the pathological condition of rheumatoid joints. However, more detailed classifications have come into use recently, ${ }^{5}$ because it is necessary to express intraarticular conditions with great accuracy. Arthrography is superior to plain radiography in providing intra-articular information.

As shown in Fig. 6, in the stage 1 group the degree of cartilaginous change is almost the same for the femoral condyles and the tibial condyles and also in the medial and lateral, which means that cartilaginous change is advancing symmetrically. The pattern of meniscal change is similar to that of the cartilage.

In the stage 1 group $16.1 \%$ of the joints showed no abnormal change in the cartilage in the medial and the lateral compartments. On the other hand in $71.4 \%$ the medial and lateral menisci were normal on the arthrograms. These results suggest that at this early stage the cartilaginous change is ahead of that of the menisci. In the stage 2 group, though the change of the cartilage, as in the stage 1 group, is symmetrical in the medial and the lateral compartments, the cartilaginous change of the femoral condyles is a little more advanced than that of the tibial condyles. The characteristic of the pathological conditions of the intra-articular components in this stage varies greatly from normal to severe, and 
PLAIN X-P (STAGE) /ARTHROGRAM

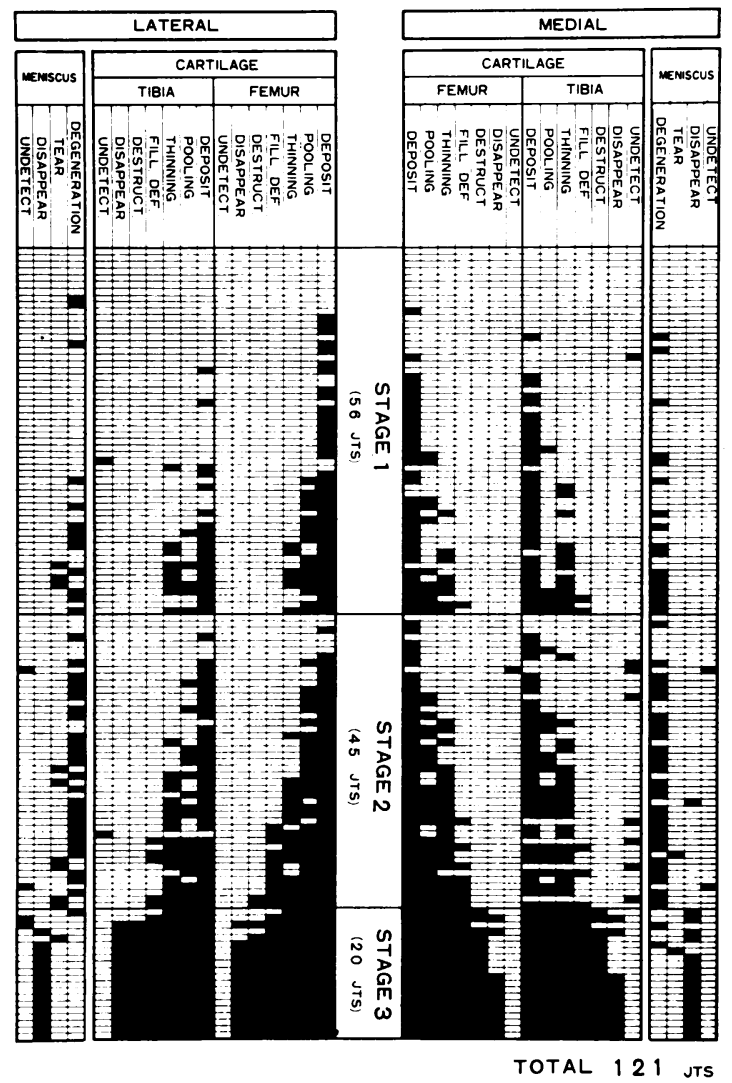

Fig. 6 Relationship between stage and changes of intra-articular components of the individual joint on arthrograms. $\square$ Indicates existence of change.

almost similar results can be seen in the menisci. In the stage 3 group $95 \%$ of the articular cartilage, $85 \%$ of the medial, and $80 \%$ of the lateral menisci showed severe change, the extent of which is similar in both compartments. From these facts it can be seen that the change in the articular cartilage is almost symmetrical through all the stages.

Despite recent research little is known about the mechanism of the progression of cartilaginous and meniscal destructive changes in the rheumatoid knee joint. As mentioned in the previous paper (Part 1), the degree and the localisation of synovial proliferation correlate closely with the pathological changes in these intra-articular components, which at least clinically begin from synovial tissue, the cartilage and menisci becoming its target. The findings that the change in the articular cartilage is a little ahead of that of the menisci in the early stage, but that the change of both compartments is almost the same in the advanced stage, are contrary to the report of Sakamoto $^{5}$ of the findings at operation, in which the meniscal changes were much more severe than those of the cartilage. It appears that rheumatoid synovial tissues are macroscopically and microscopically much more invasive to the cartilage than to the menisci.

It is necessary to appreciate not only the pathological condition of the bones of the knee joints but also that of the intra-articular components to devise more careful programmes of treatment for rheumatoid knees. The changes in the bony component in plain radiographs do not always show the changes of the intra-articular components, especially in the early stages. To fulfil this purpose arthrographic and/or arthroscopic techniques are usually used, and the former are particularly beneficial for screening because the procedure is straightforward and can be repeated on an outpatient basis.

The authors would like to thank Professor V. Wright for very helpful advice and Mrs B. Hill, who kindly corrected the English.

\section{References}

1 Andren L, Wehrin H. Double contrast arthrography of knee with horizontal roentgen ray beam. Acta Orthop Scand 1960; 39: 307-14.

2 Steinbrocker $O$. Therapeutic criteria in rheumatoid arthritis. JAMA 1949; 140: 659-62.

3 Fujikawa K, Iseki F. Arthrography of the knee joint. Clin Orthop Surg (Jpn) 1976; 11 : 216-30.

4 Fujikawa K. Arthrographic study of the rheumatoid knee. Part 1. Synovial proliferation. Ann Rheum Dis in press.

5 Larsen A. The value of individual joints for radiologic assessment of rheumatoid arthritis. Scand $J$ Rheumatol 1976; 5: 119-23.

- Sakamoto N. Clinical and radiological studies on the changes of the articular cartilages and menisci in the rheumatoid knee joints. J Jpn Orthop Assoc 1979; 53: $27-41$. 